\title{
Bioconductor caAffy Li Wong Probe Summary Method
}

National Cancer Institute

\section{Source}

National Cancer Institute. Bioconductor caAffy Li Wong Probe Summary Method. NCI

Thesaurus. Code C64223.

Summarizes probe sets using the model described in Li and Wong (2001) to a probe set with I chips and J probes. Defined in the documents available at

http://bioconductor.org/packages/1.9/bioc/html/affy.html 